\title{
Perfil da fauna de himenópteros parasitóides (Insecta, Hymenoptera) em uma área de Mata Atlântica do Parque Estadual da Fonte Grande, Vitória, ES, Brasil ${ }^{1}$
}

\author{
Celso Oliveira Azevedo ${ }^{2}$ \\ Ricardo Kawada ${ }^{2,3}$ \\ Marcelo Teixeira Tavares ${ }^{4}$ \\ Nelson Wanderley Perioto ${ }^{5}$
}

\begin{abstract}
Survey of the hymenopterous parasitoids (Insecta, Hymenoptera) from the Parque Estadual da Fonte Grande, Vitória, ES, Brazil. Parasitoids Hymenoptera were collected in a trail inside the Parque Estadual da Fonte Grande (20¹7'51.7''S; $\left.40^{\circ} 20^{\prime} 13.1^{\prime \prime} \mathrm{W}\right)$, located in an area of the Atlantic Forest, during the period of June 2000 to July 2001. A total of 4,595 specimens, belonging to 28 families, have been collected. Braconidae, Eulophidae, and Scelionidae, were the most common families, with relative abundance of $22.5 \%, 14.32 \%$, and $13.12 \%$, respectively. Sixteen families showed the relative frequency lower than $1 \%$. A preliminary list with 47 genera of some of the families is included.
\end{abstract}

KEYwords. Abundance; Atlantic Forest; Hymenoptera; parasitoids; survey.

\section{INTRODUÇÃO}

O Estado do Espírito Santo possui muitas unidades de conservação municipais, estaduais, federais e particulares e existem poucos estudos de composição faunística nessas áreas. Tais estudos, em sua maior parte, versam sobre a Estação Biológica de Santa Lúcia, no município de Santa Teresa. Brown JR. \& FreITAS (2000) encontraram 533 espécies de Lepidoptera; Simon (2000) listou 302 espécies de aves e Passamani et al. (2000) encontraram 48 espécies de mamíferos não voadores naquela localidade. AzEVEDO \& SANTOS (2000) relacionaram 30 famílias de vespas parasitóides na Reserva Biológica de Duas Bocas, no município de Cariacica, e apontaram a existência de poucos estudos sobre a composição faunística de vespas parasitóides, apesar deste grupo de insetos apresentar uma grande diversidade e ter grande importância biológica, ecológica e econômica (LASALLE \& GAULD 1991).

O objetivo desse estudo foi traçar o perfil da fauna de himenópteros parasitóides do Parque Estadual da Fonte Grande, doravante grafado como PEFG, reconhecendo as famílias presentes e sua abundância relativa, com vistas a gerar dados sobre o padrão de ocorrência e abundância relativa de famílias de vespas parasitóides em áreas de Mata Atlântica, especialmente no Espírito Santo.

\section{MATERIALEMÉTODOS}

As amostragens foram realizadas no PEFG, localizado no município de Vitória, Espírito Santo. Este parque, criado pela Lei $\mathrm{n}^{\circ} 3.875$, de 7 de agosto de 1986, é administrado pelo Instituto de Defesa Agropecuária e Florestal (IDAF) do Estado do Espírito Santo, em parceria com a Secretaria de Municipal de Meio Ambiente (SEMMAM) da Prefeitura Municipal de Vitória. O parque ocupa uma área de 217,58 hectares e fica situado no Maciço Central da Ilha de Vitória. A área é cortada pelo córrego Fradinhos e várias fontes d'água como Moscoso, Grande, São

1. Trabalho financiado pelo Fundo de Apoio à Ciência e Tecnologia (FACITEC) da Prefeitura Municipal de Vitória, ES.

2. Departamento de Biologia, Universidade Federal do Espírito Santo. Av. Marechal Campos 1468, Maruípe, 29.040-090 Vitória-ES, Brasil Endereço eletrônico: cazevedo@npd.ufes.br.

3. Bolsista de Iniciação Científica do FACITEC. Endereço eletrônico: kawada@terra.com.br.

4. Departamento de Ciências Naturais e Exatas, Centro Universitário de Araraquara. Av. Voluntários da Pátria 1309, 14.801-320 Araraquara-SP, Brasil. Endereço eletrônico: biodiversidade@uniara.com.br.

5. Instituto Biológico, Laboratório de Sanidade Animal e Vegetal de Ribeirão Preto. Rua Peru 1472-A, 14.075-310, Ribeirão Preto-SP, Brasil. Endereço eletrônico: nperioto@biologico.br. 
Benedito, Cazuza, Morcego, da gruta da Onça e Azul. A vegetação predominante é de Mata Atlântica, com alto impacto antrópico, com a presença de áreas de pasto, cultivos de banana e bambu, além da existência de habitações.

Os principais fatores de degradação do PEFG são o lixo, a coleta de mudas de plantas medicinais e ornamentais, a retirada de terra para aterros, a construção residencial, a caça, a captura de pássaros, a extração de madeira e o roubo de mourões e de arame das cercas. Além destes impactos, a população do entorno realiza atividades agrícola de subsistência e de extração mineral.

As coletas foram realizadas mensalmente, em um trecho de aproximadamente 500 metros de uma trilha de mata secundária. A trilha amostral encontra-se próxima e à esquerda da guarita de entrada do PEFG, com coordenadas geográficas $20^{\circ} 17^{\prime} 51,7^{\prime \prime} \mathrm{S}, 40^{\circ} 20^{\prime} 13,1$ "W e altitude média de 180 metros. Esta área foi escolhida por se tratar de um local com uma das vegetações menos impactadas do parque.

A amostragem foi feita através do método de varredura da vegetação, escolhido por se tratar de um dos mais eficientes na coleta de himenópteros parasitóides em geral, como indicado por Hanson \& Gauld (1995), Noyes (1989), Perioto (1991) e AzEvedo \& SAntos (2000). Nas amostragens, foi utilizada rede de varredura modelo Rose Engineering, com borda em forma de triângulo isósceles, com $36 \mathrm{~cm}$ de comprimento de lado.

Em cada coleta, foram realizados 24 ciclos amostrais de varredura, com duração de 1 minuto, desconsiderando o tempo gasto para remoção dos insetos da rede. A varredura da vegetação foi aleatória entre o nível do solo e, aproximadamente, 1,5 m acima dele, entre 10:00 e 14:00 horas.

A identificação das famílias seguiu a classificação adotada por Goulet \& Huber (1993). Foram incluídas neste estudo, todas as famílias da série Parasítica (Terebrantia) e Chrysidoidea, assim como as famílias de Vespoidea que apresentam hábito parasitóide, além daquelas que perderam, parcial ou totalmente, o hábito parasitóide. Este tratamento segue o de AzEvedo \& SANTOS (2000).

O material coletado foi depositado na Coleção Entomológica da Universidade Federal do Espírito Santo (UFES).

\section{RESULTADOS EDISCUSSÃO}

Composição das famílias. Foi amostrado um total de 4.595 exemplares de himenópteros parasitóides pertencentes a 28 famílias (Tabela 1). Foram reconhecidos também 47 gêneros na área estudada (Tabela 2). Considerando-se a existência de 61 famílias de himenópteros parasitóides no mundo e que várias destas são exclusivas de regiões zoogeográficas como a Australiana e a Holártica, verifica-se que o PEFG possui uma fauna de himenópteros parasitóides com uma diversidade razoável ao nível de família.

As famílias Aulacidae, Charipidae, Cynipidae, Embolemidae, Gasteruptiidae, Heloridae, Leucospidae, Liopteridae, Megalyridae, Ormyridae, Pelecinidae, Perilampidae, Plumariidae, Sapygidae, Sclerogibbidae, Scolebythidae, Scoliidae,
Stephanidae, Tanaostigmatidae e Trigonalyidae são relativamente escassas em coleções científicas e pouco freqüentes em amostragens de fauna de parasitóides (AzEVEDO \& SANTOS 2000), não tendo sido registradas neste estudo. Foram coletados três exemplares de Agaonidae que, em geral, são obtidos apenas em amostragens biológicas, isto é, através da coleta de sicônios de figueiras e posterior criação em laboratório.

Austronidae, Mymarommatidae, Peradeniidae, Renyxidae, Roproniidae, Rotoitidae, Tetracampidae e Vanhornidae, até o momento, não foram citadas para o Brasil, e Ibaliidae tem uma espécie introduzida (AzEvedo \& SANTOS 2000).

Foram coletadas no PEFG muitas das famílias usualmente capturadas por meio de varredura de vegetação, o que demonstra potencial de diversidade da fauna de himenópteros parasitóides neste Parque. Um fato relevante nas amostragens foi o registro das famílias Eucharitidae, Elasmidae, Tanaostigmatidae e Perilampidae, consideradas relativamente incomuns em estudos faunísticos com este método de coleta.

As famílias encontradas no PEFG, que não ocorreram na amostragem da Reserva Biológica de Duas Bocas (AzEvedo \& SAntos 2000), foram Agaonidae, Perilampidae e Tanaostigmatidae. Não foram encontradas no PEFG as famílias Figitidae, Megaspilidae, Monomachidae, Mutillidae e Proctotrupidae, que ocorrem naquela reserva. Essas oito famílias apresentam, geralmente, abundância relativa baixa, como indicado por Azevedo \& Santos (2000), Dall'Oglo et al. (2000), Noyes (1989) e Perioto (1991). Assim sendo, a ausência dessas famílias pode refletir diferenças de esforço amostral ou método de coleta e não diferenças de fauna entre as áreas. Além disso, certas famílias de vespas parasitóides como Embolemidae, Leucospidae, Pelecinidae, Perilampidae, Stephanidae são apontadas como raras e indicadoras de áreas conservadas (Aguiar 2001), mas não há estudos comparando padrões de abundância relativa de famílias de vespas parasitóides dentre diversas áreas que permitam distinguir, com clareza, em quais situações tais grupos poderiam exercer o papel de indicadores.

Abundância relativa das famílias. A abundância relativa das famílias de himenópteros parasitóides encontrados no PEFG foi a seguinte: $34,99 \%$ para Chalcidoidea (16 famílias / 1608 indivíduos); 23,48\% para Ichneumonoidea (2/1079); 20,41\% para Platygasteroidea (2/938); 10,05\% para Cynipoidea (1/462); 5,74\% para Proctotrupoidea (1/264); 2,57\% para Chrysidoidea (3/118); $2,33 \%$ para Ceraphronoidea (1/107); $0,37 \%$ para Evanioidea (1/17) e 0,04\% para Vespoidea (1/2).

As famílias Braconidae, Eulophidae, Scelionidae, Pteromalidae e Eucoilidae apresentaram a maior abundância relativa, com 1.034 indivíduos (22,50\% do total), $658(14,32 \%)$, $603(13,12 \%), 536(11,64 \%)$ e $462(10,05 \%)$, respectivamente.

A abundância de Braconidae foi maior do que a das outras famílias nas amostragens de vários meses, sendo superada por Scelionidae na amostragem do mês de novembro/2000, Eulophidae em agosto e outubro/2000, e Pteromalidae em maio/ 2001 (Tabela 1). Oito famílias ocorreram em todas as 
Tabela 1. Abundância geral das famílias de himenópteros parasitóides amostradas, por meio de varredura de vegetação, no Parque Estadual da Fonte Grande no período de julho/2000-junho/2001.

\begin{tabular}{|c|c|c|c|c|c|c|c|c|c|c|c|c|c|}
\hline FAMÍLIAS & $\mathrm{J} / 00$ & $\mathrm{~A} / 00$ & $\mathrm{O} / 00$ & $\mathrm{~N} / 00$ & $\mathrm{D} / 00$ & $\mathrm{~J} / 01$ & $\mathrm{~F} / 01$ & $\mathrm{M} / 01$ & $\mathrm{~A} / 01$ & $\mathrm{M} / 01$ & $\mathrm{~J} / 01$ & Total & $\%$ \\
\hline Ceraphronoidea & 9 & 46 & 12 & - & 4 & 9 & 1 & 2 & 12 & - & 12 & 107 & 2,33 \\
\hline Ceraphronidae & 9 & 46 & 12 & - & 4 & 9 & 1 & 2 & 12 & - & 12 & 107 & 2,33 \\
\hline Chalcidoidea & 65 & 289 & 275 & 23 & 73 & 174 & 198 & 226 & 177 & 18 & 90 & 1608 & 34,99 \\
\hline Agaonidae & 2 & - & 1 & - & - & - & - & - & - & - & - & 3 & 0,06 \\
\hline Aphelinidae & - & 14 & 8 & - & 2 & 8 & 6 & 3 & 8 & - & 2 & 51 & 1,11 \\
\hline Chalcididae & 1 & 1 & 1 & 2 & 3 & 2 & 15 & 3 & 7 & - & 3 & 38 & 0,83 \\
\hline Elasmidae & - & - & - & - & - & - & - & & 1 & - & - & 1 & 0,02 \\
\hline Encyrtidae & 8 & 51 & 28 & 1 & 4 & 18 & 16 & 7 & 9 & 1 & 18 & 161 & 3,46 \\
\hline Eucharitidae & 1 & - & 2 & - & - & 1 & - & - & - & - & - & 4 & 0,09 \\
\hline Eulophidae & 25 & 138 & 175 & 6 & 40 & 70 & 61 & 34 & 65 & 2 & 42 & 658 & 14,32 \\
\hline Eupelmidae & - & 2 & 3 & - & - & 3 & 1 & 4 & - & - & 3 & 16 & 0,35 \\
\hline Eurytomidae & 3 & 1 & 4 & 5 & 2 & 2 & 3 & 7 & 3 & - & 1 & 31 & 0,67 \\
\hline Mymaridae & - & 29 & 18 & - & - & 6 & 6 & 10 & 4 & - & 5 & 78 & 1,70 \\
\hline Perilampidae & - & - & - & - & - & - & - & 1 & - & - & - & 1 & 0,02 \\
\hline Pteromalidae & 25 & 46 & 27 & 9 & 22 & 57 & 87 & 156 & 77 & 15 & 15 & 536 & 11,64 \\
\hline Signiphoridae & - & 1 & 1 & - & - & - & 1 & 1 & 3 & - & - & 7 & 0,15 \\
\hline Tanaostigmatidae & 1 & - & 1 & - & - & - & - & - & - & - & - & 2 & 0,04 \\
\hline Torymidae & - & 2 & 1 & - & - & 3 & 2 & - & - & - & - & 8 & 0,17 \\
\hline Trichogrammatidae & - & 4 & 6 & - & - & 4 & - & 1 & - & - & 1 & 16 & 0,35 \\
\hline Chrysidoidea & 7 & 13 & 11 & 3 & 6 & 12 & 13 & 15 & 24 & 5 & 9 & 118 & 2,57 \\
\hline Bethylidae & 7 & 11 & 10 & 3 & 5 & 10 & 12 & 11 & 21 & 5 & 6 & 101 & 2,20 \\
\hline Chrysididae & - & - & - & - & 1 & - & 1 & 2 & 3 & - & - & 7 & 0,15 \\
\hline Dryinidae & - & 2 & 1 & - & - & 2 & - & 2 & - & - & 3 & 10 & 0,22 \\
\hline Cynipoidea & 45 & 90 & 82 & - & 35 & 39 & 40 & 44 & 35 & 9 & 43 & 462 & 10,05 \\
\hline Eucoilidae & 45 & 90 & 82 & - & 35 & 39 & 40 & 44 & 35 & 9 & 43 & 462 & 10,05 \\
\hline Evanioidea & 1 & 3 & 1 & - & 1 & 2 & 2 & 4 & 2 & 1 & - & 17 & 0,37 \\
\hline Evaniidae & 1 & 3 & 1 & - & 1 & 2 & 2 & 4 & 2 & 1 & - & 17 & 0,37 \\
\hline Ichneumonoidea & 89 & 136 & 103 & 10 & 69 & 162 & 142 & 138 & 127 & 17 & 86 & 1079 & 23,48 \\
\hline Braconidae & 86 & 122 & 97 & 10 & 68 & 157 & 138 & 137 & 122 & 17 & 80 & 1034 & 22,50 \\
\hline Ichneumonidae & 3 & 14 & 6 & - & 1 & 5 & 4 & 1 & 5 & - & 6 & 45 & 0,98 \\
\hline Platygasteroidea & 56 & 226 & 172 & 19 & 72 & 88 & 76 & 65 & 86 & 8 & 70 & 938 & 20,41 \\
\hline Platygasteridae & 9 & 99 & 79 & 7 & 13 & 31 & 18 & 20 & 35 & 3 & 21 & 335 & 7,29 \\
\hline Scelionidae & 47 & 127 & 93 & 12 & 59 & 57 & 58 & 45 & 51 & 5 & 49 & 603 & 13,12 \\
\hline Proctotrupoidea & 50 & 107 & 26 & 4 & 12 & 18 & 10 & 10 & 16 & 2 & 9 & 264 & 5,74 \\
\hline Diapriidae & 50 & 107 & 26 & 4 & 12 & 18 & 10 & 10 & 16 & 2 & 9 & 264 & 5,74 \\
\hline Vespoidea & - & - & - & 2 & - & - & - & - & - & - & - & 2 & 0,04 \\
\hline Tiphiidae & - & - & - & 2 & - & - & - & - & - & - & - & 2 & 0,04 \\
\hline Total de indivíduos & 322 & 910 & 682 & 61 & 272 & 504 & 482 & 504 & 479 & 60 & 319 & 4595 & 100 \\
\hline Total de famílias & 17 & 21 & 24 & 11 & 16 & 21 & 20 & 22 & 19 & 10 & 18 & 28 & 100 \\
\hline Cumulação famílias & 17 & 24 & 24 & 25 & 26 & 26 & 26 & 27 & 28 & 28 & 28 & 28 & 100 \\
\hline
\end{tabular}

amostragens e outras sete famílias não ocorreram apenas em uma ou duas amostragens. Algumas foram mais expressivas em determinadas coletas, como os Ceraphronidae no mês de agosto/2000, com 46 em 107 indivíduos coletados, o que representa $43 \%$ do total, e Diapriidae com 40,5\% deles coletados no mês de agosto/2000.

As famílias Aphelinidae, Bethylidae, Ceraphronidae, Diapriidae, Encyrtidae, Mymaridae e Platygastridae apresentaram abundância que variou entre $7,29 \%$ e $1,11 \%$ do total amostrado (Tabela 1). Contribuição inferior a 1\%, isto é, 46 espécimes, foi registrada para 16 das 28 famílias, a saber
Agaonidae, Chalcididae, Chrysididae, Dryinidae, Elasmidae, Eucharitidae, Eupelmidae, Eurytomidae, Evaniidae, Ichneumonidae, Perilampidae, Signiphoridae, Tanaostigmatidae, Tiphiidae, Torymidae, Trichogrammatidae, com percentuais que variaram entre $0,98 \%$ a $0,04 \%$, o que é pouco representativo nessa área.

De maneira geral, as amostragens foram prejudicadas pelo regime de chuvas durante o estudo pois, apesar da varredura de vegetação ser um dos métodos mais eficientes de coleta de vespas parasitóides, é dependente de vegetação seca. Não houve coleta em setembro/2000, devido às chuvas; a coleta de 
Tabela 2. Lista preliminar de gêneros reconhecidos no Parque Estadual da Fonte Grande.

\begin{tabular}{|c|c|}
\hline Famílias & Gêneros \\
\hline Aphelinidae & Encarsia Förster \\
\hline \multirow[t]{5}{*}{ Chalcididae } & Brachymeria Westwood \\
\hline & Dirhinus Dalman \\
\hline & Haltichella Spinola \\
\hline & Melanosmicra Ashmead \\
\hline & Notaspidium Dalla Torre \\
\hline Elasmidae & Elasmus Westwood \\
\hline Eucharytidae & Kapala Cameron \\
\hline \multirow[t]{6}{*}{ Eulophidae } & Astichus Förster \\
\hline & Chrysocharis Förster \\
\hline & Euplectrus Westwood \\
\hline & Galeopsiomyia Girault \\
\hline & Pediobius Walker \\
\hline & Purisminus Walker \\
\hline \multirow[t]{3}{*}{ Eupelmidae } & Aracnophaga Ashmead \\
\hline & Reikosiella Yoshimoto \\
\hline & Anastatus Motshulsky \\
\hline \multirow[t]{2}{*}{ Eurytomidae } & Bephratelloides Girault \\
\hline & Eurytoma Illiger \\
\hline \multirow[t]{4}{*}{ Encyrtidae } & Anagyrus Howard \\
\hline & Emencyrtus Ashmead \\
\hline & Encyrtus Latreille \\
\hline & Zeteticontus Silvestri \\
\hline Perilampidae & Perilampus Latreille \\
\hline \multirow[t]{2}{*}{ Pteromalidae } & Erotolepsia Howard \\
\hline & Lelaps Haliday \\
\hline Signiphoridae & Signiphora Ashmead \\
\hline Tanaostigmatidae & Tanaostigma Howard \\
\hline \multirow[t]{2}{*}{ Torymidae } & Palmon Dalman \\
\hline & Podagrion Spinola \\
\hline \multirow[t]{6}{*}{ Bethylidae } & Anisepyris Kieffer \\
\hline & Apenesia Westwood \\
\hline & Dissomphalus Ashmead \\
\hline & Goniozus Förster \\
\hline & Pseudisobrachium Kieffer \\
\hline & Rhabdepyris Kieffer \\
\hline \multirow[t]{2}{*}{ Dryinidae } & Aphelopes Dalman \\
\hline & Neodryinus Perkins \\
\hline \multirow[t]{2}{*}{ Chrysididae } & Adelphe Mocsáry \\
\hline & Amesiga Cameron \\
\hline Eucoilidae & Odonteucoila Ashmead \\
\hline \multirow[t]{3}{*}{ Evaniidae } & Evaniella Bradley \\
\hline & Hyptia Illiger \\
\hline & Semaeomyia Bradley \\
\hline Scelionidae & Baryconus Förster \\
\hline
\end{tabular}

novembro foi interrompida por uma chuva brusca após terem sido amostrados apenas 4 minutos, e a coleta de maio/2001 foi realizada com vegetação muito úmida, devido também à chuvas. Mesmo assim, foram coletados 18,83 parasitóides por minuto de varredura, superior ao coletado por NOYEs (1989) com este mesmo método (14,89 himenópteros parasitóides coletados/ minuto), mas inferior àquele obtido por AzEvEdo \& SANTOS
(2000) (28,84 himenópteros parasitóides coletados/minuto). Quando desconsideramos as amostragens de novembro/2000 e de maio/2001, prejudicadas pelas chuvas, a média de vespas parasitóides por minuto de coleta passa a ser de 20,72.

No estudo de PerIOTO (1991), os Mymaridae foram os mais abundantes, com $23,75 \%$ do total amostrado. Scelionidae, Braconidae e Eucoilidae, com 6,26\%, 5,41\% e 0,75\% do total amostrado, foram as sexta, sétima e $19^{\mathrm{a}}$ famílias mais abundantes, respectivamente. Logo, a principal diferença dos dados de Perioto (1991) é a alta abundância relativa de Mymaridae, o que pode estar relacionado com os métodos de coleta, uma vez que nesse estudo foram utilizados cinco outros métodos, além de ter sido conduzido em uma área de cerrado.

Foram coletadas, no PEFG, cerca de 18 famílias de himenópteros parasitóides por amostragem e, com apenas as duas primeiras coletas, a cumulação de famílias atingiu 24 das 28 totais coletadas, isto é $85,7 \%$ (Tabela 1 ).

Dall'Oglio et al. (2000) encontraram 2.099 de himenópteros parasitóides pertencentes a 26 famílias em uma área de eucaliptal no município de Ipaba, Minas Gerais, utilizando 11 armadilhas Malaise durante um ano. Isto representa apenas 0,52 himenópteros parasitóides por dia de amostragem por armadilha. Tais dados, tomados em agroecossistemas, apresentam diversidade e abundância menores de himenópteros parasitóides, mesmo quando comparados com a área silvestre impactada aqui estudada.

De maneira geral, o padrão de abundância relativa das famílias de vespas parasitóides encontrado no PEFG foi semelhante àquele observado na Reserva Biológica de Duas Bocas, desconsiderando-se as famílias encontradas apenas em uma das áreas. Considerando-se o presente estudo e aquele realizado por AzEVEDO \& SANTOS (2000) observou-se que o uso de vespas parasitóides no estudo de comunidades animais em áreas de Mata Atlântica, discriminou áreas com diferentes graus de impacto mais pela diferença no número de famílias coletado do que por diferenças no padrão de suas abundâncias relativas.

Agradecimentos. Ao Edson Val Passos, da Secretaria Municipal de Meio Ambiente da Prefeitura Municipal de Vitória (SEMMAM), pela liberação do PEFG para as coletas e pelo apoio e incentivo; ao guardaparque Joadir Luiz Ganda, pela segurança e apoio durante as coletas; ao Fundo de Apoio à Ciência e Tecnologia da Prefeitura Municipal de Vitória (FACITEC) pelo suporte financeiro e bolsa de iniciação científica.

\section{REFERÊNCIAS}

Aguiar, A. P. 2001. Manual de informações sobre conservação e vida silvestre de insetos de Mata Atlântica. São Paulo, publicação autônoma, 100p., 49 figs.

Azevedo, C. O. \& H. S. Santos. 2000. Perfil da fauna de himenópteros parasitóides (Hymenoptera) em uma área de Mata Atlântica da Reserva Biológica de Duas Bocas, Cariacica, ES, Brasil. Boletim do Museu de Biologia Mello Leitão 11/12:117-126.

Brown JR., K. S. \& A. V. L. Freitas. 2000. Diversidade de Lepidoptera em Santa Teresa, Espírito Santo. Boletim do Museu de Biologia Mello Leitão 11/12:71-116.

Dall'Oglio, O. T.; J. C. Zanuncio; C. O. Azevedo \& A. G. B. Medeiros. 2000. Survey of the Hymenoptera parasitoids in Eucalyptus grandis 
in a native vegetation area in Ipaba, State of Minas Gerais, Brazil. Anais da Sociedade Entomológica do Brasil 29(3): 583-588.

Hanson, P. E. \& I. D. Gauld (eds.). 1995. The Hymenoptera of Costa Rica. Oxford, Oxford University Press, xii $+893 p$.

Goulet, H. \& J. T. Huber (eds.). 1993. Hymenoptera of the World: An identification guide to families. Ottawa, Agriculture Canada Publication, vii $+668 \mathrm{p}$.

Lasalle, J. \& I. D. Gauld. 1991. Parasitic Hymenoptera and the biodiversity crisis. Redia 74(3): 315-334.

Noyes, J. S. 1989. A study of five methods of sampling Hymenoptera (Insecta) in a tropical rainforest, with special reference to the Parasitica. Journal of Natural History 23: 285-298.
Passamani, M.; S. L. Mendes \& A. G. Chiarelo. 2000. Non-volant mammals of the Estação Biológica de Santa Lúcia, and adjancent areas of Santa Teresa, Espírito Santo, Brazil. Boletim do Museu de Biologia Mello Leitão 11/12: 215-228.

Perioto, N. W. 1991. Perfil da Fauna de Hymenoptera Parasitica, incluindo Chrysidoidea, do Cerrado da Fazenda Canchim (EMBRAPA, São Carlos, SP). Dissertação de Mestrado, UFSCar, São Carlos, vi +70 p.

Simon, J. E. 2000. Composição da avifauna da Estação Biológica de Santa Lúcia, Santa Teresa, Espírito Santo. Boletim do Museu de Biologia Mello Leitão 11/12: 149-170. 